\title{
Advances in clinical molecular imaging instrumentation
}

\author{
Brian F. Hutton $^{1} \mathbb{D} \cdot$ Kjell Erlandsson ${ }^{1} \cdot$ Kris Thielemans $^{1}$
}

Received: 4 December 2017 / Accepted: 11 January 2018 / Published online: 31 January 2018

(c) The Author(s) 2018. This article is an open access publication

\begin{abstract}
In this article, we describe recent developments in the design of both single-photon emission computed tomography (SPECT) and positron emission tomography (PET) instrumentation that have led to the current range of superior performance instruments. The adoption of solid-state technology for either complete detectors [e.g., cadmium zinc telluride (CZT)] or read-out systems that replace photomultiplier tubes [avalanche photodiodes (APD) or silicon photomultipliers (SiPM)] provide the advantage of compact technology, enabling flexible system design. In SPECT, CZT is well suited to multi-radionuclide and kinetic studies. For PET, SiPM technology provides MR compatibility and superior time-of-flight resolution, resulting in improved signal-to-noise ratio. Similar SiPM technology has also been used in the construction of the first SPECT insert for clinical brain SPECT/MRI.
\end{abstract}

Keywords Positron emission tomography $\cdot$ Single-photon emission computed tomography $\cdot$ Hybrid imaging $\cdot$ Solid-state detectors · Small-field detectors

\section{Introduction}

Despite major advances in technology in the past 40 years, the basic design of imaging instrumentation has remained relatively unchanged. The most commonly used instrument in clinical nuclear medicine is the gamma camera, first developed by Hal Anger in 1958 [1]. This instrument with an array of photomultiplier tubes (PMTs) that converts the light produced by interaction of gamma radiation with a scintillation crystal continues to be widely used for planar imaging and single-photon emission computed tomography (SPECT). Performance of these systems continues to be dominated by the need for physical collimation. Positron emission tomography (PET) has traditionally involved similar design principles with most systems using block detectors, operating in coincidence, which effectively act as small gamma cameras, again based on PMT read-out of scintillation light (albeit without the need for physical collimation). Historically, there have been many novel designs introduced, particularly for organ-specific imaging [2], but few if any of these have stood the test of time. It is only recently that

Brian F. Hutton

b.hutton@ucl.ac.uk

1 Institute of Nuclear Medicine, University College London, London, UK alternative technologies have made an impact on the clinical marketplace. This recent evolution opens new opportunities for future molecular imaging.

In this article, recent advances in technology that are influencing the current design of molecular imaging instrumentation are described. Examples of commercially available systems that utilise these technologies (including small-field cameras, SPECT, and PET) are given, with some insight to the motivation of these designs and the potential benefits that they provide. Finally, a brief perspective on future potential development is given.

\section{Recent developments in detector technology}

Sodium iodide $[\mathrm{NaI}(\mathrm{Tl})]$ continues to be the main scintillator used for gamma cameras having superior light output compared to alternative materials, ideally suited to singlephoton imaging. Several detector materials have offered promise as candidates for future systems: e.g., lanthanum bromide $(\mathrm{LaBr})$ has sufficiently high stopping power and good light output. However, there have been problems in growing large crystals; $\mathrm{LaBr}$ is hygroscopic (like $\mathrm{NaI}$ ) and also emits intrinsic radiation. Most commercial PET systems use lutetium oxyorthosilicate (LSO) or lutetium yttrium 
orthosilicate (LYSO) due to the attractive timing properties of these materials that supports time-of-flight (TOF) estimation. Nevertheless, bismuth germinate (BGO) is still used as an inexpensive option, albeit with no TOF capability. An overview of new scintillator materials can be found in [3].

The main development in detector design has involved the adoption of solid-state technology. This involves two distinct types of technology: solid-state detectors that provide direct conversion of gamma photon energy to an electronic signal, effectively replacing the more conventional scintillator and PMT combination; and solid-state read-out systems that replace the PMTs but still rely on scintillation crystals. Both have the advantage over the conventional technology of being compact, which opens opportunity for novel designs and specific applications. Readers are referred to [4] for a useful technical overview.

\section{Solid-state detectors}

The solid-state detector is not a new technology [5], but its late introduction to mainstream clinical imaging has probably been the result of relatively high cost. The two most commonly used detector materials are Cadmium Zinc Telluride (CdZnTe or CZT) or, less commonly, Cadmium Telluride (CdTe). In these detectors, the interaction of a gamma ray with the detector produces ionisation with free electrons/holes attracted by an applied bias voltage so as to produce a measurable charge, proportional to the absorbed photon energy. Detectors are designed with small elements or pixels (usually isolated on one electrode with a common electrode opposite), so that spatial localisation is based on simply identifying the element where the signal originates. In clinical practice, the elements are usually approximately $2.5 \mathrm{~mm} \times 2.5 \mathrm{~mm}$ square, which defines the intrinsic resolution of the system, but smaller elements are certainly feasible. The final instrument can be very compact and in applications where the camera can be placed close to the activity source, so as to minimise the influence of collimator resolution, the system resolution is superior to that of a conventional camera. A further advantage is the full use of the detection area, unlike the use of Anger logic where the edge area of the detector is usually excluded due to positioning inaccuracies. To isolate the signal to a single pixel, the detector thickness is usually limited to a few millimetres with, as a result, limited stopping power (especially at higher energies). Note that as pixel size is reduced the thickness must also be reduced and, as a consequence, stopping power or sensitivity is further reduced. The sensitivity to $140 \mathrm{keV}$ photons of ${ }^{99 \mathrm{~m}} \mathrm{Tc}$ is of the order of $70 \%$ for a $5 \mathrm{~mm}$-thick CZT detector compared to almost $100 \%$ for the $9.5 \mathrm{~mm}$-thick sodium iodide crystals coupled to PMTs usually used in a conventional gamma camera. These detectors are, therefore, not ideal for use with PET, since the fraction of $511 \mathrm{keV}$ annihilation photons that are stopped is low.

A definite advantage of CZT is the superior energy resolution compared to a conventional gamma camera (typically around 5.5\% for available commercial systems compared to 9-10\%). This aids in energy discrimination and so in theory is ideally suited for dual radionuclide studies. A closer inspection of the typical energy spectrum, however, reveals a significant tail on the photopeak (see Fig. 1), which results mainly from charge diffusion where the signal arising from photon interaction is shared in neighbouring elements. Steps can be taken to reduce this effect by detecting and summing coincident neighbouring signals. However, in most commercial systems, the tail results in a significant contribution to the acquired counts that must be accounted for when dealing with spillover (down-scatter from a higher energy radionuclide) or scatter correction. In clinical practice, the tail can typically contribute $\sim 50 \%$ of counts in a scatter window
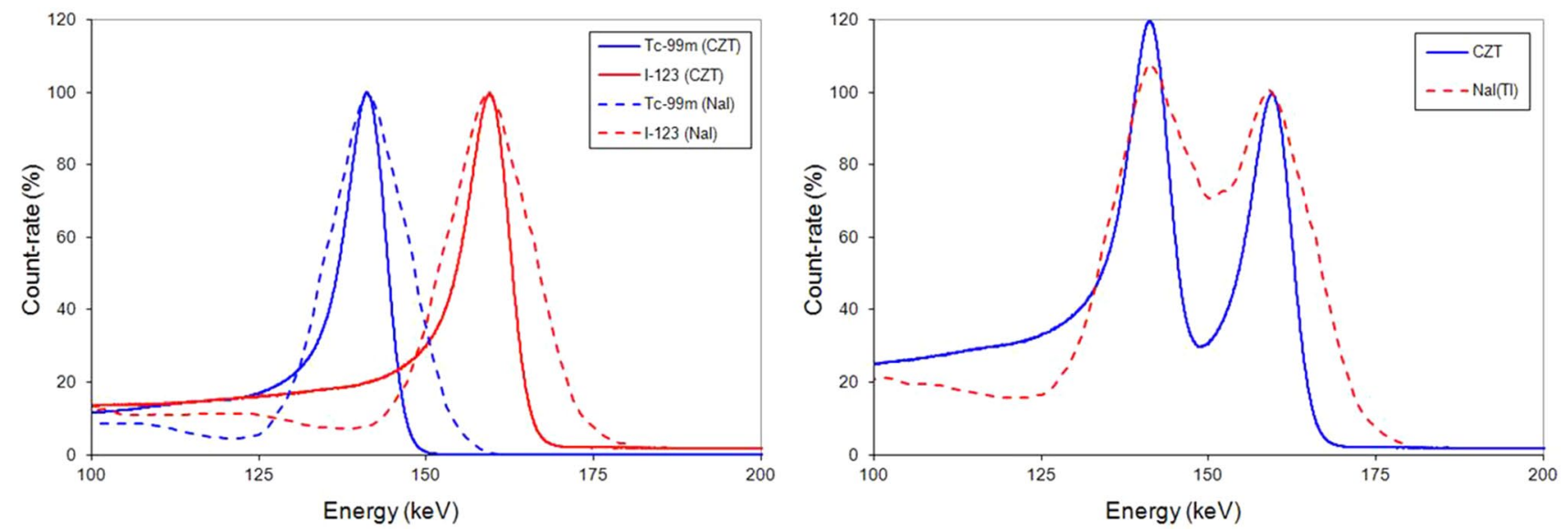

Fig. 1 CZT energy spectra: Illustrative CZT spectra (left) for ${ }^{99 \mathrm{~m}} \mathrm{Tc}$ and ${ }^{123} \mathrm{I}$ for CZT and NaI(Tl) detectors. The spectra for the combined dual radionuclides illustrate the improved discrimination using CZT (right) 
placed below the photopeak, necessitating the adoption of complex multi-window methods when dealing with correction for scatter or spillover [6-8].

Until recently, use of CZT in imaging instruments has been limited to hand-held surgical cameras and small organspecific imaging systems, where the cost of populating the detector volume is less prohibitive. Only recently has a more conventional sized gamma camera been released based on CZT technology (GE Healthcare, 2016) (Fig. 2).

A further technology that has been explored is that of charge coupled devices (CCD), the devices used to capture photographs on compact cameras or telephones. These have been used in conjunction with image intensifiers to directly record the light output from a scintillation crystal or phosphor, achieving very high intrinsic resolution. These can be used for high-resolution digital autoradiography [9]. Although a preclinical camera has been designed using similar technology [10], to date, this approach has not been adapted for clinical SPECT use.

\section{Solid-state read-out devices}

In contrast to solid-state detectors that replace both scintillator and PMTs, solid-state read-out devices are used to convert light emitted from a scintillator to an electrical signal, effectively replacing the PMTs. These devices share the advantage of being well suited to applications that require a compact system design. Like semiconductor detectors, these devices have undergone development over several decades, but the recent strong interest in applying the technology in molecular imaging arose largely due to the fact that, unlike the PMT, these devices are insensitive to a magnetic field and so are well suited to hybrid imaging of PET or SPECT and MRI. They can be combined with dense scintillators that are optimal for the detection of $511 \mathrm{keV}$ annihilation photons. The devices used in the first clinical PET/MRI systems introduced by Siemens Healthcare were avalanche photodiodes (APDs) [11], but more recently, silicon photomultipliers (SiPMs) [12] have been used. A brief outline of the principles and properties of these devices follows.

APDs consist of a light-sensitive layer where light photons produce electron-hole pairs (photo-electric effect), with thickness of the layer chosen to match the wavelength emitted from the scintillator. Application of a high electric field of several volts per micrometre accelerates the electrons with the production of secondary ionisation (avalanche) and a resulting amplified electrical signal, although the gain is significantly less than with the conventional PMTs. A limitation of the APD is the relatively slow rise time of the signal, which renders the device unsuitable for time-of-flight PET detection. The device also needs to be operated at a stable temperature. If the applied reverse-bias voltage is increased sufficiently, then the APD operates in Geiger mode (in analogy to a Geiger counter). The device can then provide a large signal in response to a single light photon and is referred to as a single-photon avalanche diode (SPAD), which can be manufactured as a very small device $(20-100 \mu \mathrm{m})$. A Silocon PM is an array of SPADs, read-out in parallel to provide a signal proportional to the number of detected light photons in a small detector area (cell). The photo-detection efficiency is similar to a conventional PMT, and importantly, the gain is also similarly large $\left(\sim 10^{6}\right)$, while using a much smaller operating voltage than required by the conventional PMT. An array of SiPMs is then used in place of the PMT array
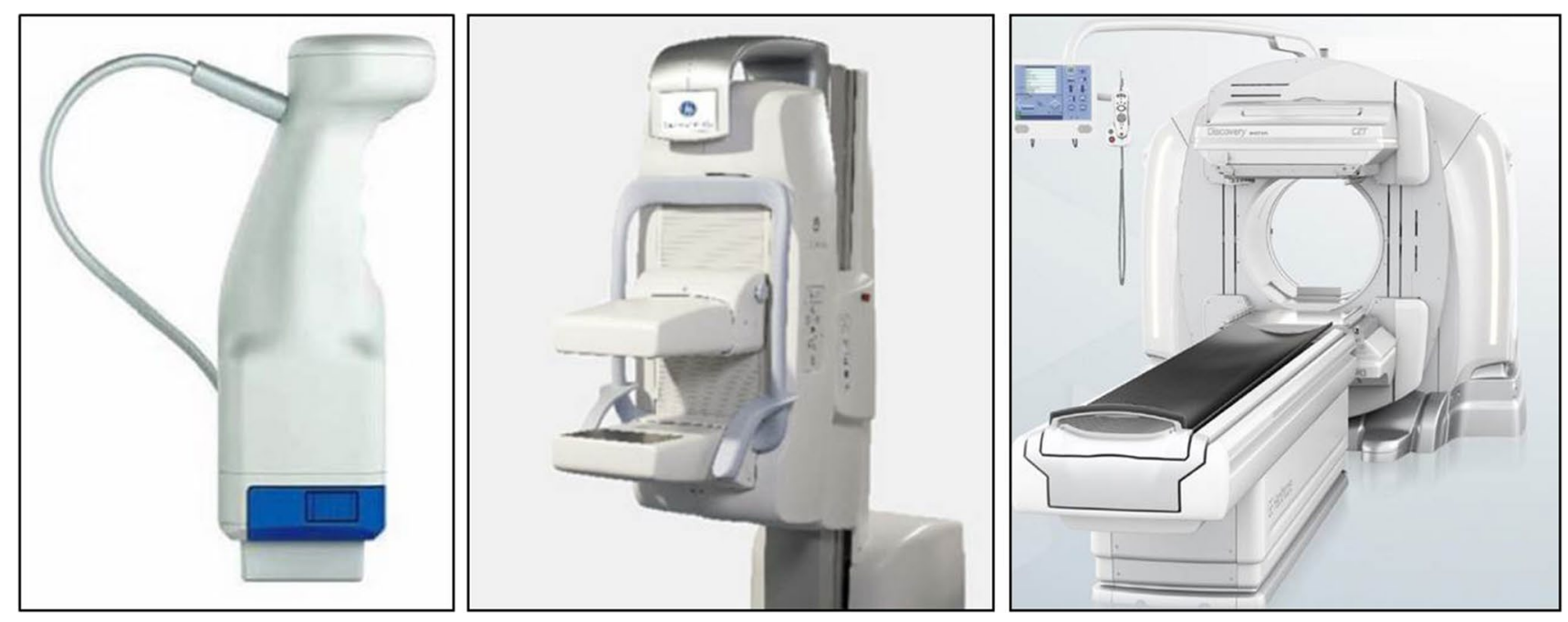

Fig. 2 Range of CZT imaging devices: CZT has been used in a range of devices including small hand-held imaging probes for intrasurgical imaging (GE Healthcare) (middle) and culminating with the release of a standard size gamma camera (GE Healthcare) (right) 
to enable positioning and energy estimation based on the detected light distribution (Fig. 3). A significant advantage of SiPMs is the superior timing resolution which enables time-of-flight (TOF) estimation [13]. TOF time resolution less than 100 ps has been demonstrated in bench-top experiments [14] and, at the time of writing, the first results of a prototype commercial PET system based on LSO have been presented with a TOF timing resolution of $250 \mathrm{ps}$ [15], superior performance to the most recent PMT-based systems. Further improvement in timing resolution is to be expected as the technology develops [16]. As in the case of APDs, SiPMs are virtually insensitive to magnetic fields. They do also need to operate at a stable temperature and noise is reduced if cooling is adopted. A digital SiPM has also been developed by Philips [17, 18]; this incorporates read-out electronics with an array of SiPMs to provide position and energy signals as a direct digital output avoiding the need for independent spatial decoding.

\section{Detection position estimation}

Until recently, most commercial PET scanners were based on block detectors with an array of long, narrow crystals connected to an array of read-out devices (e.g., PMTs or SiPMs) to identify the crystal in which the interaction took place. The point of interaction at depth within the crystal is, however, unknown. This leads to resolution degradation due to the parallax effect, which increases with increasing distance from the centre of the field-of-view [19]. This problem can be addressed by measuring the depth-of-interaction (DOI) in the crystals. Various ways of doing this have been proposed, mainly based on solid-state detectors for scintil- utilise a depth-dependent light-sharing between two crystals [22]. With the exception of the Siemens HRRT scanner [23], no DOI estimation is being used in standard commercial scanners.

A further alternative method is to use a dual read-out approach, with light sensors at both ends of the crystal. The relative signal from the two sensors gives an estimate of the DOI. This was initially proposed by Moses et al. [24] using photodiodes at one end and PMTs at the other end of the crystals. Later, the same principle was implemented using position-sensitive APDs at both ends [25, 26]. Recently, several novel approaches, based on light-sharing between crystals and SiPM read-out, have been proposed. In one, a 3D array of crystals is completely surrounded by SiPMs [27]. Two approaches use light-sharing in orthogonal directions at top and bottom of the crystals with either single-ended SiPM read-out [28], or separate $\mathrm{x}$ - and $\mathrm{y}$-read-out on the sides of the crystals [29]. Yet, another approach uses a light-guide and reflector at the top of the crystal-array and SiPM readout at the bottom [30]. It is not clear at this point which of these approaches might be adopted by camera manufacturers in the future.

In contrast to PET scanners, gamma cameras and SPECT systems usually use monolithic crystals coupled to an array of PMTs (with exception of CZT systems). A 2D position of interaction is usually obtained by a simple centroid calculation based on the signal from the different PMTs [1]. However, a better spatial resolution and linearity can be obtained using a maximum-likelihood (ML) position estimation [31, 32]. Normally, 2D positioning is considered sufficient, but DOI information can be useful, especially if non-parallelhole collimators are used [33,34]. Recently, the interest for

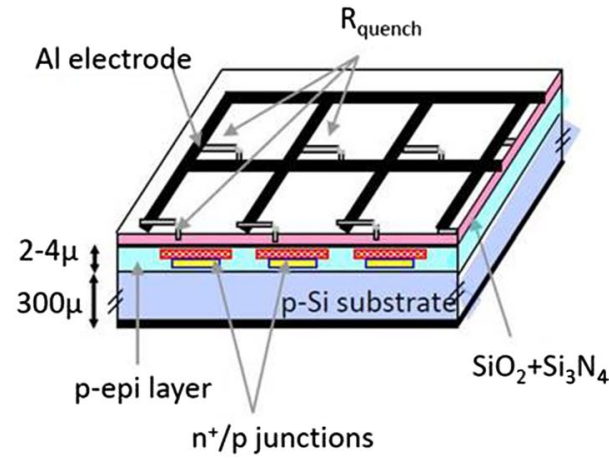

Fig. 3 SiPM principle: SiPM tiles contain several thousand singlephoton APDs operating in Geiger mode, with an output signal for the tile that is proportional to the total number of detected light photons.

lation light read-out. The most straightforward approach is to use multiple layers of crystals that can be identified based on differences in the pulse shape for light emitted from different types of crystals [20, 21]. Another possibility is to
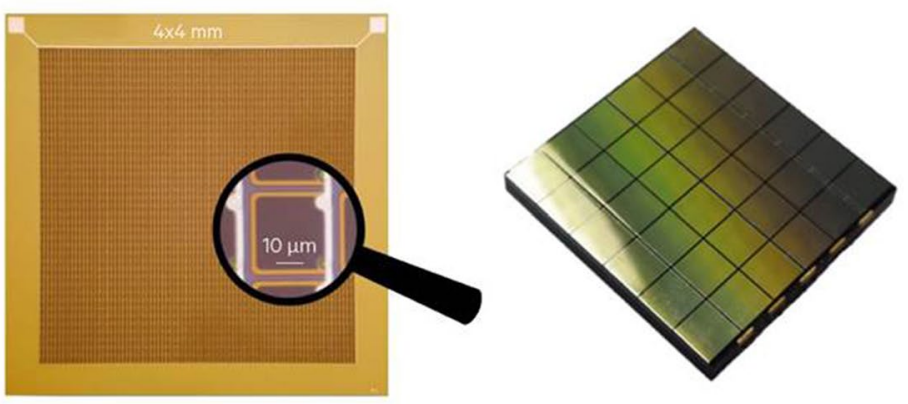

The light distribution detected by an array of tiles then provides the information to determine location and energy of the detected gamma photon

using monolithic crystals in PET has been increasing and DOI estimation algorithms have been developed for this purpose [35-37]. A novel algorithm based on machine learning was recently presented by Pedemonte et al. [38]. 


\section{Recent developments in imaging instruments}

\section{Small-field planar gamma cameras/imaging probes}

Small hand-held planar gamma cameras have been designed for use in surgery to aid in the identification of either sentinel nodes or active tumours [39]. The identification of the sentinel node has become a widespread technique in surgery where it is important to establish that disease is localised, with low probability of spread through the lymph system; identification of the lymph nodes that are located on the drainage path associated with the tumour site avoids the need for harvesting of the complete lymph system, which can have serious side effects. The use of a camera as opposed to a simple probe system has obvious advantage in localisation of the active volume. The image can be combined with an optical camera so as to further inform the surgical team [40]. Clearly, the camera needs to be sufficiently light and compact so as to not impede surgery and so that it can be easily manipulated. CZT technology is clearly well suited to this application.

Several groups have developed CZT-based intrasurgical systems and there are a range of cameras commercially available. Small-field planar gamma cameras have been developed (e.g., for thyroid imaging), although few have survived as viable commercial systems. This has probably been dictated by the limited demand for dedicated systems that require a large referral rate to justify their use. The one area of application that continues to be of interest is scinti-mammography [41]. There have been several reports of commercial CZT-based systems (e.g., marketed by GE Healthcare) with the current dual head models providing compelling clinical evidence of superior lesion detection [42]. The proximity of the detectors to the patient, in the form of breast paddles that are used for mild compression, ensures optimal resolution for this system.

\section{SPECT}

There are several examples of solid-state cameras being used for dedicated SPECT systems, specifically designed for use in cardiac imaging (Fig. 4). The D-SPECT (Spectrum Dynamics) takes advantage of the compact detector design to provide a novel detector geometry where multiple small detectors rotate on their axes to acquire multiple projections from a predefined field-of-view $[43,44]$. The organ-specific acquisition is combined with wide-beam collimation to enhance the acquired counts from the heart region, determined from a scout view; as a result, acquisition time can be shortened compared to conventional SPECT (by a factor of 3-4). Each of the nine detectors is constructed using an array of $16 \times 64 \mathrm{CZT}$ elements (each $2.46 \mathrm{~mm} \times 2.46 \mathrm{~mm}$ ). GE has used similar CZT technology [45, 46], instead using 19 detector arrays of $32 \times 32$ CZT elements, each stationary detector equipped with a single pinhole collimator directed to a common volume where the patient's heart needs to be sited by physically moving the patient (GE NM530c). The system provides similar gain in acquisition time to the D-SPECT. A variation on the D-SPECT design principle for more general application has been announced by Spectrum Dynamics and will be marketed in 2018 as the Veriton (www.spectrum-dynamics.com) (Fig. 5). In this case, a full ring of 12 detectors, each rotating on their axes, is used with detectors positioned so as to be close to the patient (including movement of selected detectors so as to be close to the head for brain imaging). The flexible design opens opportunity for flexible organ-specific acquisition providing a capability for 'adaptive' SPECT (a term originally introduced by Barrett et al. [47] to describe task-dependent acquisition).

As stated earlier, scinti-mammography can provide useful information in cases where the conventional X-ray mammography results are inconclusive. SPECT can provide additional information, as compared to planar scintigraphy. A compact CZT-based gamma camera was used to develop a dedicated mammography SPECT scanner [48]. The compact size of the camera allows for the use of complex $3 \mathrm{D}$
Fig. 4 Dedicated cardiac SPECT systems: two examples of CZT-based dedicated cardiac SPECT systems are the Spectrum Dynamics D-SPECT (left) and the GE NM530c multipinhole-based system (right)
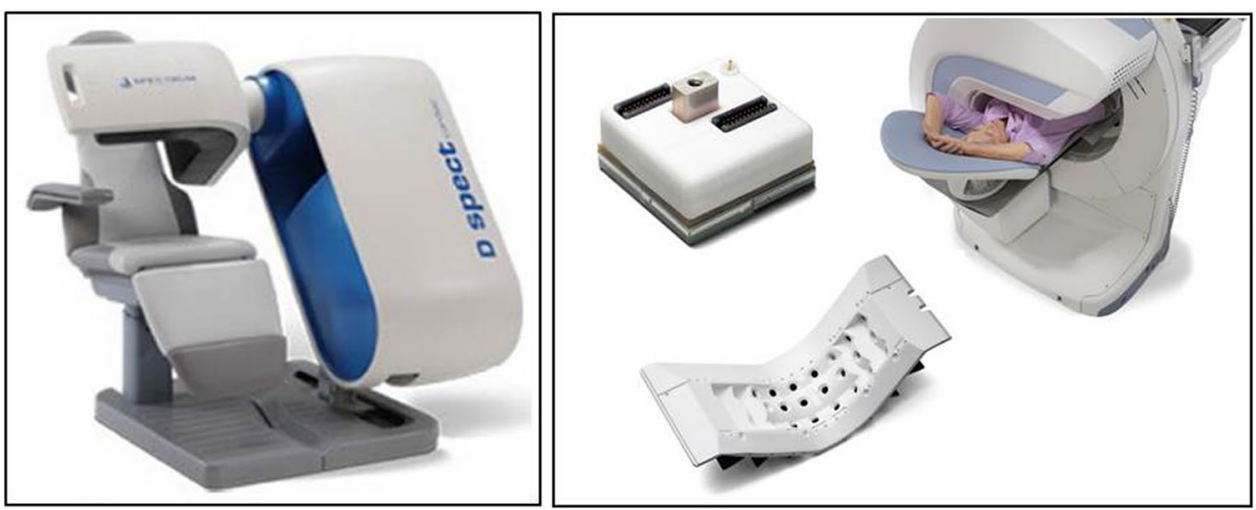


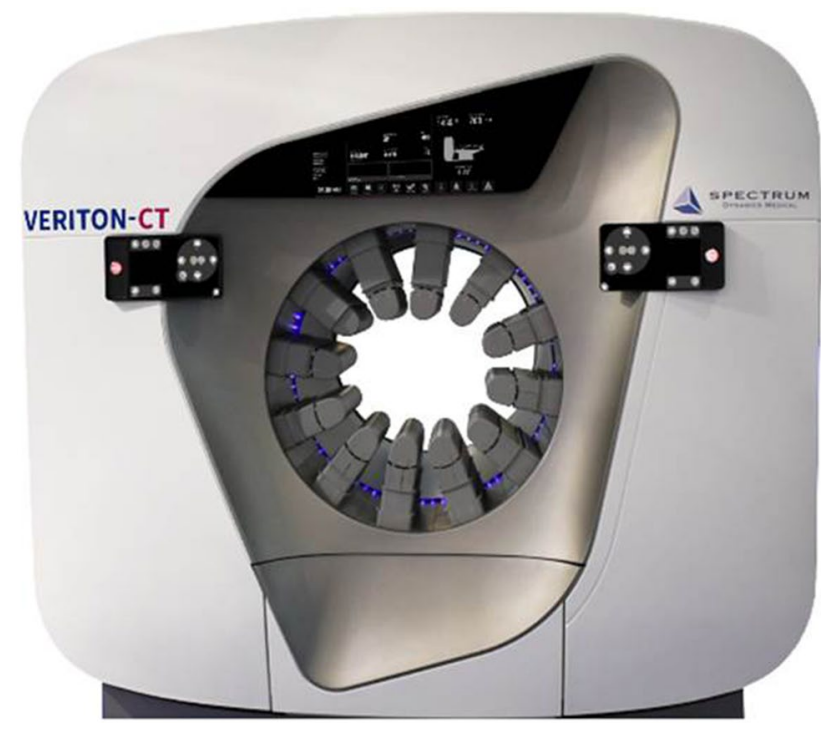

Fig. 5 Whole-body adaptive SPECT: the Veriton system from Spectrum Dynamics enables adaptive acquisition from a selected region as well as tight contouring by moving individual detectors close to the patient

acquisition orbits with polar camera tilt, to optimise the field-of-view of the system.

The overall performance of a gamma camera (resolution vs. sensitivity) tends to be dominated by the collimation used. In the conventional gamma cameras, usually parallelhole collimators are used with dimensions chosen to suit the photon energy for the radionuclide of interest. In contrast, in preclinical systems, pinhole collimation is used to magnify the field-of-view with a corresponding improvement in system resolution. This results from the improvement in the intrinsic component of the system resolution by a factor equal to the magnification. This principle can be used to provide sub-millimetre resolution, ideal for imaging of small rodents, but has also recently formed the basis for systems available for clinical brain imaging. A similar approach is adopted in the G-SPECT brain imaging system being promoted by MILabs where resolution of less than $3 \mathrm{~mm}$ has been demonstrated [49]. In this case, the field-of-view is smaller than the brain and requires movement of the patient bed so as to capture information from the complete head (Fig. 6). However, with improvement in the intrinsic resolution of detectors, pinhole collimation can be used with a different objective, placing the pinhole aperture closer to the detector so as to result in minification rather than magnification. Using this approach, multiple small compact detectors can be used to achieve a significant gain in sensitivity while maintaining resolution $[50,51]$. Slit-slat collimators provide a compromise, adopting parallel-hole collimation along the axial direction so as to preserve the useful field-of-view but pinhole (slit) collimation in the transaxial direction. This type of collimator is considered optimal for imaging the human brain [52].

As an alternative to the gain in sensitivity that can be achieved with multi-pinhole collimators in combination with high intrinsic resolution detectors, the goal can be to achieve a more compact design, well suited to applications where mobility is important or space limited. This has been exploited in the design of a compact MRI-compatible SPECT system which enables simultaneous SPECT/MRI imaging of a patient's head (Fig. 7). This system is based on caesium iodide scintillators coupled to SiPM read-out, customised to the wavelength of the scintillation light produced. MRI-compatible electronics provides on-board positioning logic with optical fibre connection to a more remote data acquisition system [53]. The detector has been designed to provide $1 \mathrm{~mm}$ intrinsic resolution, well suited to compact design. The device is equipped with a novel multi-aperture slit-slat collimator [54] that provides minification, while maintaining slat length, to provide a stationary SPECT acquisition, a necessary feature of an MR-compatible system. This stationary system has the added advantage of facilitating dynamic acquisition, avoiding the problems of rotation inherent in the conventional SPECT systems. Demonstrating feasibility with a demountable head-only insert [55] may stimulate interest in developing whole-body SPECT/MRI, following a similar development pathway to PET/MRI. The challenges in achieving compatibility with
Fig. 6 High-resolution brain SPECT: the MiLabs G-SPECT system sequentially images small regions with magnification, to cover the complete volume occupied by the brain. The excellent spatial resolution achieved $(<3 \mathrm{~mm})$ is superior to that obtained with conventional SPECT

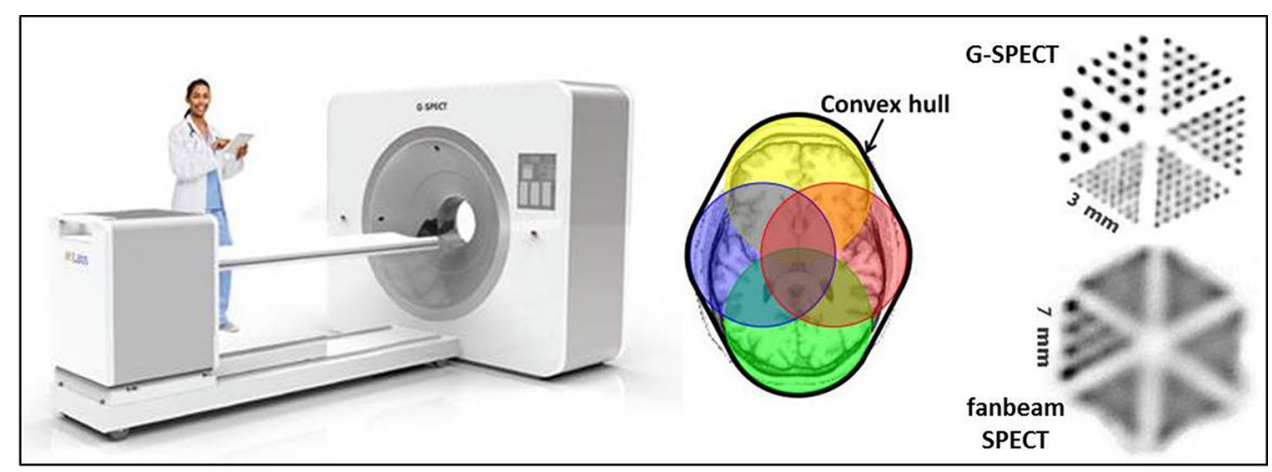



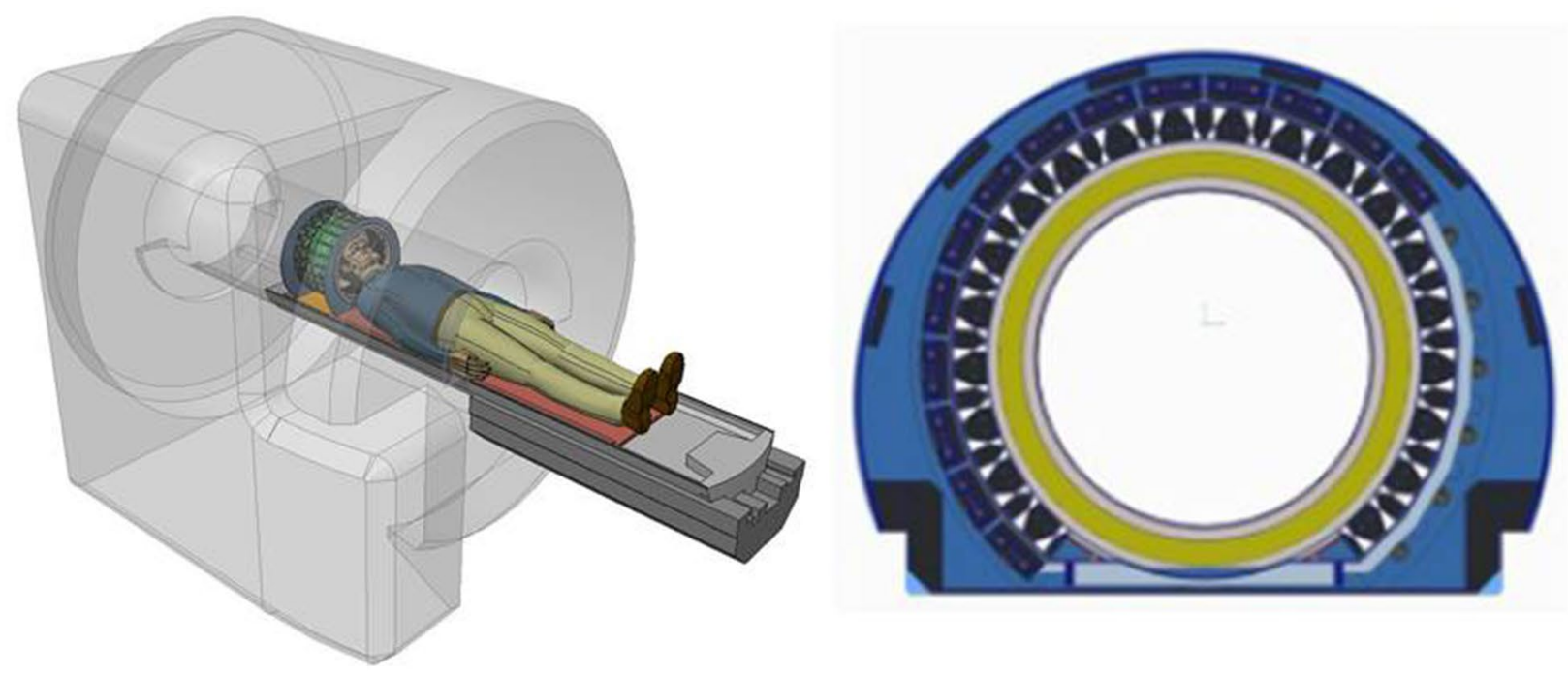

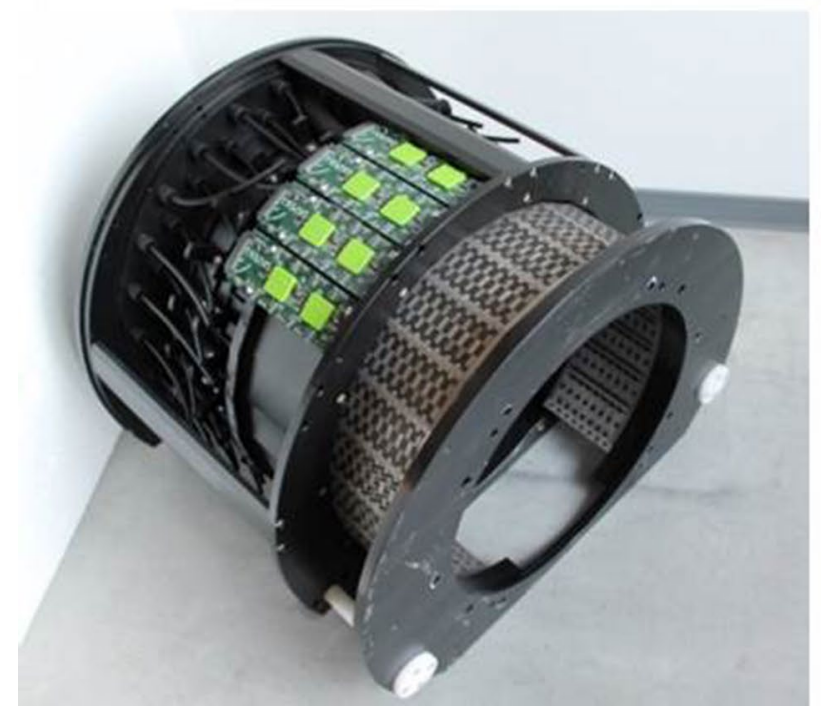

Fig. 7 SPECT/MRI insert: An MRI-compatible SPECT insert uses high intrinsic resolution detectors with SiPM read-out in combination with a multi-mini slit-slat collimator to achieve similar performance

MRI are similar to those encountered in PET/MRI, with the added possibility of introducing eddy currents in the collimator. The availability of additive manufacturing technology [56] can aid in the manufacture of complex collimators that achieve effective radiation shielding while introducing divided components that minimise the possibility of eddy currents [57].

\section{PET}

Most PET systems have been designed for general wholebody scanning, however, like SPECT, there can be advantage in designing organ-specific systems. Reducing the detector ring diameter for brain or breast imaging can result in improvement of both sensitivity (increased detection solid

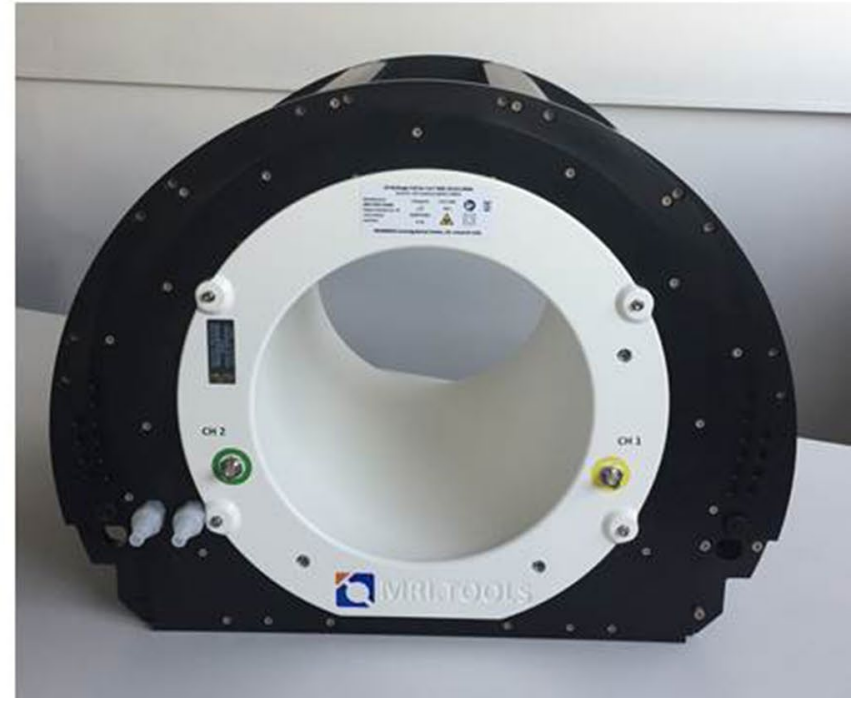

to a conventional dual head SPECT system. A transmit/receive head coil is fitted between the detector and patient aperture

angle) and resolution (reduced effect of annihilation photon non-colinearity). Note, however, that DOI effects become significant for these systems and so DOI information is important. There has been renewed interest in developing dedicated brain systems. Systems with resolution less than $2 \mathrm{~mm}$ are being developed, benefiting from combined TOF and DOI capability. In addition, the adoption of monolithic crystals rather than block detectors is leading to improved spatial resolution [58]; an example is the recently introduced compact CareMiBrain (www.oncovision.com) (Fig. 8). Several groups have also developed wearable brain imaging systems that are supported on the subject's head so as to enable acquisition during free movement of the subject [59-61]. These clearly depend on availability of compact, light-weight technology. Positron Emission Mammography 
(PEM) has been also proved to be a valuable complement to the conventional X-ray mammography for detection and staging of breast cancer, and a number of different PEM systems have been developed over the years [62]. These systems evolved from stationary dual-headed systems, based on the limited-angle tomography principle, to rotating and full-ring systems, providing fully tomographic images. For example, the ClearPEM scanner is a rotating dual-headed system, based on LYSO crystals sandwiched between two APD arrays, providing DOI information with a spatial resolution of $\sim 1.3 \mathrm{~mm}$ [63]. Excellent TOF timing resolution will also enable new designs which would otherwise suffer from limited-angle artefacts, in PEM systems [64], but also in in-beam PET for proton radiotherapy [65].

Historically, the design of clinical PET/CT systems was fairly stable, mainly based on block detectors and PMT readout [66]. As mentioned above, the development of simultaneous PET/MRI has acted as a stimulus for technological development [67-69]. The need for compact, MR-compatible components has stimulated the fast adoption of solidstate read-out systems, initially APDs, but more recently SiPMs. The superior coincidence timing has resulted in a better TOF timing resolution than was previously achievable, with the result that similar PET designs are being adopted for the latest PET/CT instruments $[15,70,71]$. Prior to the introduction of SiPM-based PET, the coincidence timing resolution in commercial systems was in the order of $600 \mathrm{ps,}$ with a signal-to-noise gain of around 1.8 for a $30 \mathrm{~cm}$ object. In the most recent systems, the timing resolution is much improved with increasing evidence of improved clinical performance (Fig. 9) [72]. Further gains are likely as detector performance continues to improve.

Apart from the technical challenges related to compatibility issues, another challenge in PET/MRI systems is the attenuation correction, as the MRI image cannot be used directly to create a transmission map. A number of different

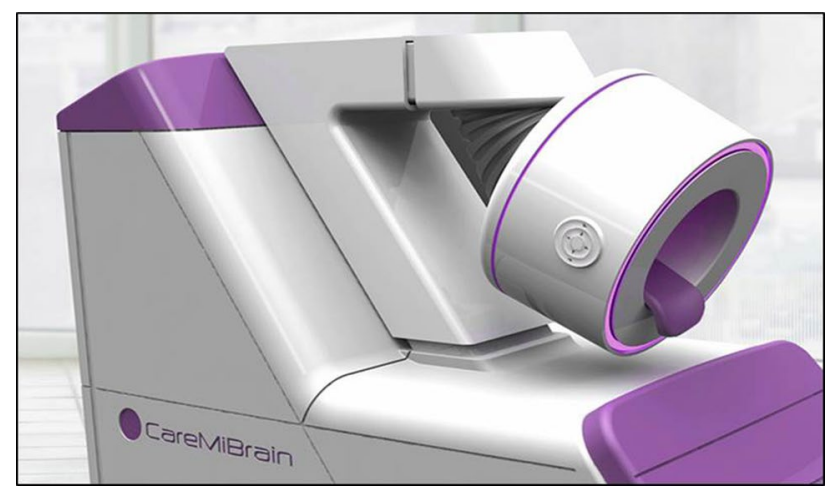

Fig. 8 Dedicated brain PET: novel designs for dedicated brain PET are appearing on the market with compact systems such as the illustrated system from OncoVision which has better than $2 \mathrm{~mm}$ spatial resolution approaches have been developed to address this problem [73], particularly directed to achieving quantitative brain PET reconstruction. However, in the case of the thorax, where attenuation is patient and disease specific, work is still in progress. On the other hand, PET/MRI has several advantages over PET/CT. There is no additional radiation dose with MRI and MRI provides better soft-tissue contrast, especially in brain and pelvic studies. MRI can be used for motion tracking, for estimation of multiple complementary parameters using different pulse sequences and for estimation of physiological parameters using dynamic MRI imaging following injection of a contrast agent,.

There is increasing interest in designing extended axial length PET systems that would enable whole-body imaging (Fig. 10). This work has been pioneered by the EXPLORER consortium [74], but there are already several additional research groups considering similar designs. The development is motivated by possible applications, e.g., whole-body kinetic studies which may be of interest in pharmaceutical development; significantly improved sensitivity leading to ultra-low-dose whole-body PET imaging which might open avenues for paediatric applications and screening programs. The systems are likely to be expensive due to the high volume of detectors and they raise further technological issues, e.g., the need to determine DOI and to deal with the anticipated high scatter fraction.

\section{Recent developments in reconstruction software}

Although the focus of this article is on instrumentation it is clear that the developments in image reconstruction continue to play an integral role in achieving optimal image quality for both SPECT and PET. It is the combination of instrument capability and use of appropriate algorithms that provides the final images that are clinically interpreted. A good example is the case of TOF PET imaging where the signal-to-noise gains are a direct consequence of the additional information available from coincidence timing during reconstruction.

Iterative reconstruction [75] has become the standard approach, providing flexibility in handling detector geometry, straightforward incorporation of measured attenuation, and opportunity to include details of detector resolution and estimated motion. There is increasing evidence that extending reconstruction to handle not just the three-dimensional activity distribution but also changes that occur in time due to motion or tracer kinetics can provide improved image quality. Although maximum-likelihood reconstruction based on the ordered subsets expectation maximization (OSEM) algorithm [76] is widely used, there is growing recognition that alternative algorithms can provide advantage (reduced 
Fig. 9 Whole-body PET images comparing the Siemens SiPM-based prototype Biograph Vision with $<250$ ps TOF resolution (right) with their $<540$ ps TOF resolution (left). Lesions can be better identified due to the improved contrast with improved TOF resolution. Note that differences in imaging start time (60 vs. $113 \mathrm{~min}$ post-administration) and reconstruction parameters may partly account for the observed differences in image quality (Data courtesy of University of Tennessee and Siemens) conventional mCT system with
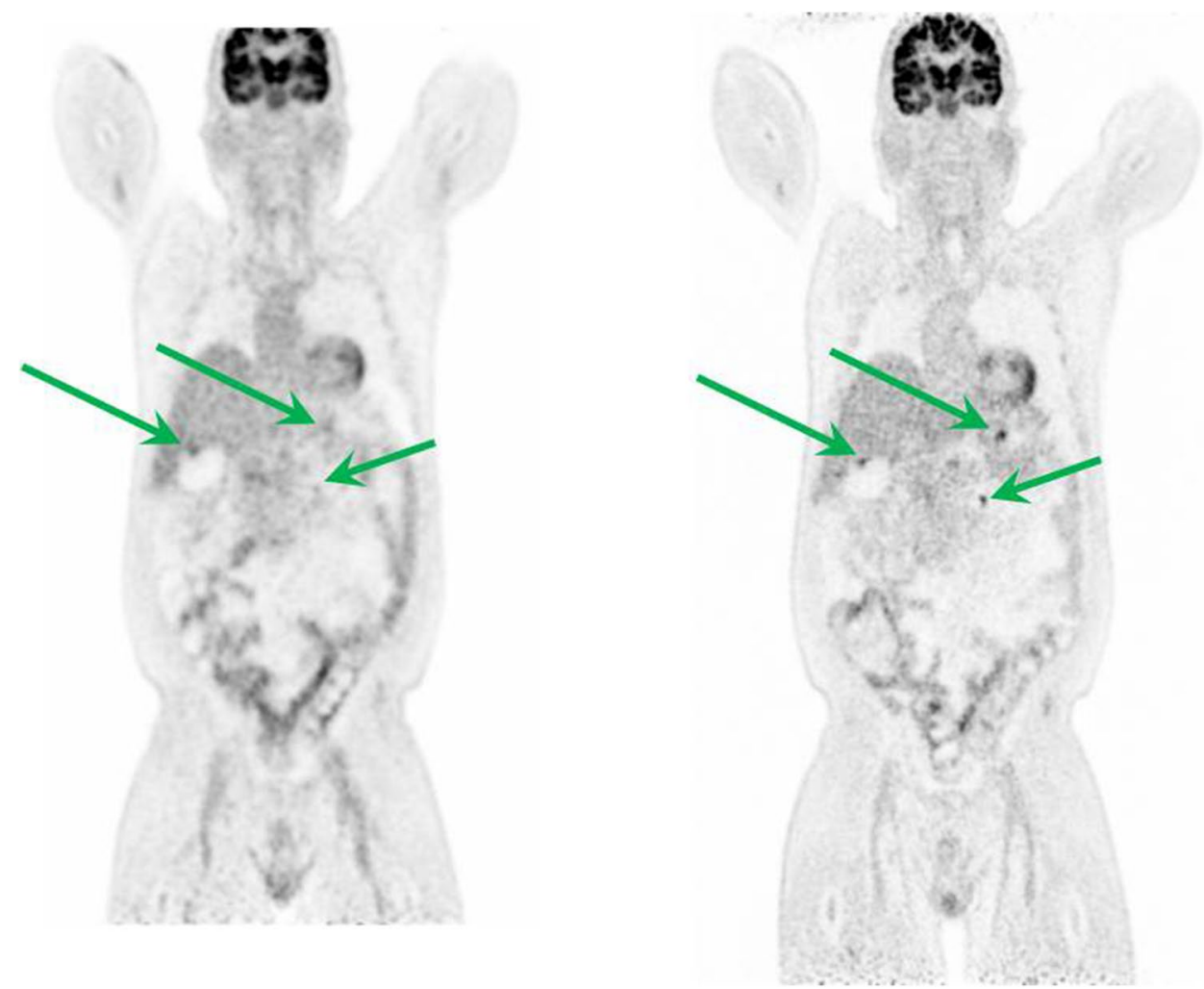

Fig. 10 Explorer-whole-body PET: a major development led by the team at UC Davis in conjunction with United Imaging. The group is developing a whole-body PET system which will acquire a whole-body PET with a 40 times gain in sensitivity compared to current systems. Illustrated are the conceptual design (left) and mock system (right)
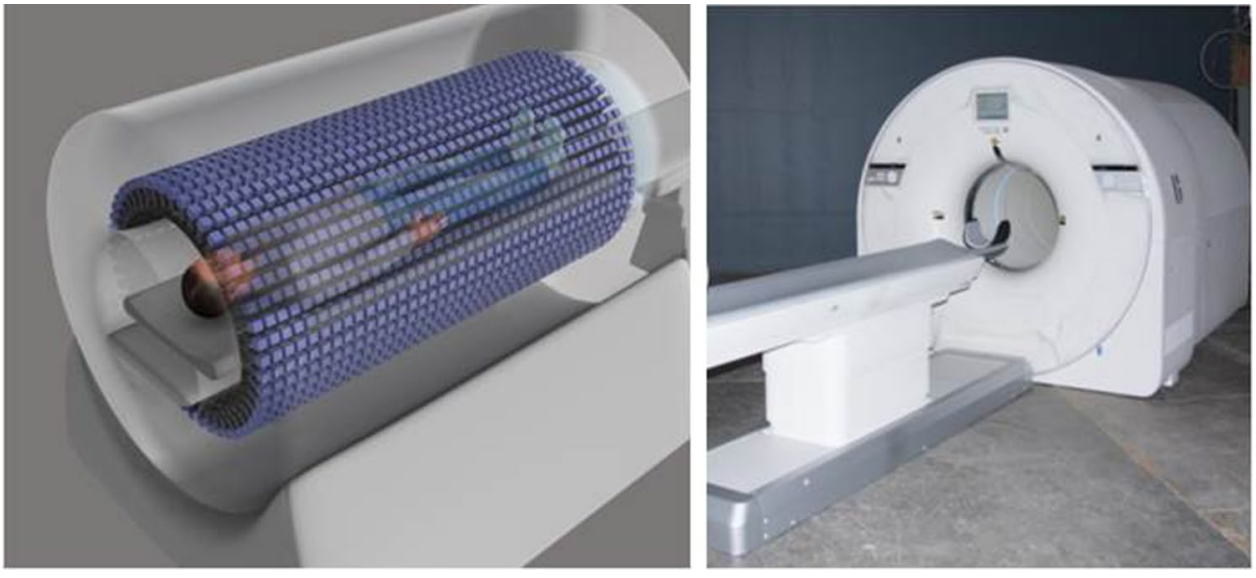

bias and better precision) with acceleration strategies that enable practical use. Some strategies are not new but have taken considerable time to gain acceptance, taking advantage of the ever-increasing capabilities of modern computers. Vendors are faced with a dilemma in choosing from a growing range of possible approaches, where choice of parameters is often poorly understood by end-users and full validation rarely available. Standardisation across systems becomes increasingly challenging as suppliers add customised solutions to tackle noise control and computational efficiency. This is further complicated by the need to select task-specific solutions.
A development, which has led to improved spatial and temporal accuracy, was the introduction of list-mode reconstruction $[77,78]$. In this case, the detection coordinates and other relevant information are stored for each detected event during acquisition. The main advantage of the latter approach is that it makes it possible to utilise the most accurate information supported by the scanner hardware in terms of position, energy, TOF information, or time; as in the traditional histogram format, data size can become impractical. 


\section{Noise control}

The problem of noise in PET and SPECT images is normally addressed by some kind of regularisation technique, such as low-pass filtering to reduce the intensity of high-frequency components. An alternative approach that can be used in iterative algorithms is to incorporate the concept of a "likely image" as a priori information during the reconstruction process, so that the algorithm is guided towards a solution with a low noise level. This is known as a Bayesian or Maximum A Posteriori (MAP) algorithm. One example of a prior is the Relative Difference penalty [79] which penalises large relative differences between neighbouring voxel values, but with the option to have some edge preservation. This penalty forms the basis for the recent Q.Clear commercial implementation [80]. Just like other regularisation methods, MAP introduces extra parameters. It is still a topic of research on how to set optimal parameters for a particular task (such as lesion detection [81]).

Regularisation can lead to a reduction in the spatial resolution. However, it is possible to compensate for this by utilising high-resolution anatomical information from CT or MRI images as a priori information during the reconstruction (for reviews, see [82, 83]). Alternatively, a postreconstruction partial volume correction (PVC) procedure can be used (for reviews, see [83, 84]). These techniques are made more accessible by the introduction of multi-modality systems (PET or SPECT combined with CT or MRI), reducing the need for image co-registration (for recent reviews, see [55, 85-87]). These techniques also open the door for methods in which PET data are used to improve the quality of MRI images [88].

\section{Time-of-flight}

In recent years, commercial PET scanners capable of timeof-flight (TOF) measurement have been introduced (for recent review, see [13]). By measuring the time-difference between the detection of the two annihilation photons, the annihilation position along the LOR can be estimated, leading to improved signal-to-noise ratio (SNR), as well as faster convergence. Other benefits of TOF were, perhaps, less expected but are just as important. These include increased robustness to errors in the attenuation map [89-91] and the possibility to estimate the attenuation map (or the attenuation factors) from the emission data [92, 93]. They all follow from the increased stability of the reconstruction problem. With the novel solid-state detector technology, the increased TOF resolution is expected to improve the benefits of TOF dramatically. As mentioned previously, TOF information can improve image quality in systems with restricted geometry such as partial-ring or dual-headed PEM [64].

\section{Accounting for temporal changes}

There is sometimes a need to correct for patient motion, including body motion, respiratory, and cardiac motion. The correction can be either rigid or non-rigid, and requires either dynamic or gated data acquisition. The motion-pattern can be estimated directly from the data, or can be obtained using an external motion-tracking device or MRI data. Motion correction can be incorporated in the reconstruction process [94-96].

Dynamic acquisition also allows for kinetic analysis of the data [97, 98], which can provide additional information that could be clinically relevant, by analysing the uptake and washout of the tracer as a function of time. Kinetic analysis and image reconstruction can be performed independently; however, combining the two procedures into a 4D parametric reconstruction algorithm can lead to improved results [99] (for reviews, see [100-103]). Motion correction is fundamental for parametric imaging, and a method for estimating both motion and kinetic parameters simultaneously was recently presented [104]. With combined PET/MRI systems, it is possible to develop kinetic models that utilise both dynamic PET and dynamic MRI data for parameter estimation [105]. These systems can also be useful for non-invasive estimation of the input function, which is normally required for kinetic modelling [106].

A recent development that is of practical interest is the possibility to acquire whole-body PET kinetics by repeatedly scanning the patient through multiple bed positions or through repeated continuous axial motion of the patient. The resulting set of time frames can be used in conjunction with a population-based input function to derive kinetic parameters (e.g., via Patlak analysis). Since the set of acquisition times differs for each transaxial slice, the $4 \mathrm{~d}$ reconstruction software needs to include additional timing information, so that parametric images can be produced for the whole-body.

Dynamic acquisition has always been problematic using SPECT due to the detector rotation that is normally necessary. With the advent of stationary systems (e.g., GE NM530c and the SPECT/MRI insert) continuous dynamic acquisition similar to that obtained in PET is feasible [107]. This opens the potential for extraction of tracer kinetic parameters and the possibility to use radiopharmaceuticals whose uptake is not necessarily constant over time (usually a necessary condition for conventional SPECT).

As the dimensionality of the data increases, with the acquisition of dynamic and/or gated data from multiple modalities, the increased availability of fast data processing hardware, such as the Graphical Processing Unit (GPU), makes it possible to implement reconstruction much faster than previously possible. This also opens possibility to perform computationally demanding tasks such as bootstrap uncertainty estimation [108]. 


\section{Conclusions and future prospects}

Imaging continues to play an important role in patient management and has an increasingly relevant role in precision medicine where individuals can benefit from personalised treatment. The search for better detectors continues, although the adoption in commercial systems tends to be relatively slow, partly due to cost considerations but also due to the need for stable temperature-independent performance and robust production methods. The research community continues to play an important role in exploring the potential application of new technologies to medical imaging, and optimising the quality of images that are produced and the robustness of parameters that can be extracted. In this article, there is an emphasis on the recent adoption of solid-state technology in the development of both SPECT and PET systems. The system designs that are appearing offer potential to support novel future applications and to stimulate the extended use of nuclear medicine procedures. In addition, the compact design that is achievable with solidstate technology would be well suited to SPECT systems with small footprints that can be installed in specialist clinics or designed as mobile systems that can be used at the patient's bedside, a capability formerly only possible with small planar cameras.

As the capability of instrumentation continues to evolve it should be borne in mind that the ultimate design should be defined by clinical need, not simply by engineering invention. Changes in instrument capability can, however, influence development in radiochemistry, e.g., opening possibility for tracers suitable for dynamic SPECT studies rather than limiting the choice of tracer to those which reach equilibrium, as required for rotating SPECT acquisition.

The rapid development of solid-state read-out devices continues to reduce the barriers for PET TOF imaging, with theoretical limits for timing resolution now well below $100 \mathrm{ps}$. This is leading to significant gains in signal-to-noise ratio in addition to potential reduction of certain artefacts and limitations. The suppliers recognise this potential and already are moving towards the adoption of SiPM technology as an option across their range of PET instruments.

There continues to be some debate regarding the likely future demand for the hybrid option of PET/MRI, with the capital cost hard to justify in the clinical setting. There are now a significant number of systems in tertiary institutions and research centres, and there may still be unforeseen applications that drive this market in the future. There also continues to be uncertainty regarding the future demand for organ-specific systems. Throughout the history of imaging instrument development, there have been many designs for use in dedicated studies of brain, heart, and breast, with evidence that superior performance can be achieved for specific tasks. In general, however, there is more interest in systems that provide flexible application, particularly as clinical demands change over time. System designs that provide optimal flexibility in acquisition protocols are certainly desirable.

Acknowledgements This work was partly supported by the UK National Institute of Health Research, University College London Hospitals, Biomedical Research Centre.

Author contributions BFH: literature search and review; manuscript writing. KE: literature search, manuscript writing and editing. KT: manuscript writing and editing.

\section{Compliance with ethical standards}

Conflict of interest Brian Hutton, Kjell Erlandsson, and Kris Thielemans have received research support from GE Healthcare, Siemens, and Spectrum Dynamics, and have collaborated with Mediso Medical Imaging Systems, Nuclear Fields, FBK, MRI.tools, and PETsys Electronics.

Open Access This article is distributed under the terms of the Creative Commons Attribution 4.0 International License (http://creativeco mmons.org/licenses/by/4.0/), which permits unrestricted use, distribution, and reproduction in any medium, provided you give appropriate credit to the original author(s) and the source, provide a link to the Creative Commons license, and indicate if changes were made.

\section{References}

1. Anger HO (1958) Scintillation camera. Rev Sci Instrum 29(1):27-33. https://doi.org/10.1063/1.1715998

2. Hutton BF (2014) The origins of SPECT and SPECT/CT. Eur J Nucl Med Mol Imaging 41(Suppl 1):S3-S16. https://doi. org/10.1007/s00259-013-2606-5

3. Lecoq P (2016) Development of new scintillators for medical applications. Nucl Instr Meth Phys Res A 809(Supplement C):130-139. https://doi.org/10.1016/j.nima.2015.08.041

4. Peterson TE, Furenlid LR (2011) SPECT detectors: the Anger Camera and beyond. Phys Med Biol 56(17):R145-R182. https ://doi.org/10.1088/0031-9155/56/17/R01

5. Wagenaar DJ (2004) CdTe and CdZnTe semiconductor detectors for nuclear medicine imaging. In: Wernick MNAJ (ed) Emission tomography: the fundamentals of SPECT and PET. Elsevier, San Diego, pp 269-291

6. Kacperski K, Erlandsson K, Ben-Haim S, Hutton BF (2011) Iterative deconvolution of simultaneous $99 \mathrm{mTc}$ and $201 \mathrm{Tl}$ projection data measured on a CdZnTe-based cardiac SPECT scanner. Phys Med Biol 56(5):1397-1414. https://doi. org/10.1088/0031-9155/56/5/012

7. Holstensson M, Erlandsson K, Poludniowski G, Ben-Haim S, Hutton BF (2015) Model-based correction for scatter and tailing effects in simultaneous $99 \mathrm{mTc}$ and 123I imaging for a CdZnTe cardiac SPECT camera. Phys Med Biol 60(8):3045-3063. https ://doi.org/10.1088/0031-9155/60/8/3045

8. Fan P, Hutton BF, Holstensson M, Ljungberg M, Pretorius PH, Prasad R, Ma T, Liu Y, Wang S, Thorn SL, Stacy MR, Sinusas AJ, Liu C (2015) Scatter and crosstalk corrections for (99m)Tc/ (123)I dual-radionuclide imaging using a CZT SPECT system 
with pinhole collimators. Med Phys 42(12):6895-6911. https:// doi.org/10.1118/1.4934830

9. Miller BW, Barber HB, Barrett HH, Wilson DW, Chen L A (2006) Low-cost approach to high-resolution, single-photon imaging using columnar scintillators and image intensifiers. In: 2006 IEEE nuclear science symposium conference record, Oct. 29 2006-Nov. 1 2006, pp 3540-3545. https://doi.org/10.1109/ nssmic.2006.353763

10. Miller BW, Barber HB, Furenlid LR, Moore SK, Barrett HH (2009) Progress of BazookaSPECT, pp 74500C-74500C-74515

11. Shah KS, Farrell R, Grazioso R, Harmon ES, Karplus E (2002) Position-sensitive avalanche photodiodes for gamma-ray imaging. IEEE Trans Nucl Sci 49(4):1687-1692. https://doi. org/10.1109/TNS.2002.801510

12. Dolgoshein B, Balagura V, Buzhan P, Danilov M, Filatov L, Garutti E, Groll M, Ilyin A, Kantserov V, Kaplin V, Karakash A, Kayumov F, Klemin S, Korbel V, Meyer H, Mizuk R, Morgunov V, Novikov E, Pakhlov P, Popova E, Rusinov V, Sefkow F, Tarkovsky E, Tikhomirov I (2006) Status report on silicon photomultiplier development and its applications. Nucl Instr Meth Phys Res A 563(2):368-376. https://doi.org/10.1016/j.nima.2006.02.193

13. Vandenberghe S, Mikhaylova E, D'Hoe E, Mollet P, Karp JS (2016) Recent developments in time-of-flight PET. EJNMMI Phys 3(1):3. https://doi.org/10.1186/s40658-016-0138-3

14. Schaart DR, van Dam HT, Seifert S, Vinke R, Dendooven P, Lohner H, Beekman FJ (2009) A novel, SiPM-array-based, monolithic scintillator detector for PET. Phys Med Biol 54(11):35013512. https://doi.org/10.1088/0031-9155/54/11/015

15. Casey M, Burbar Z, Rothfuss H, Panin V, Bharkhada D, Howe W, Bradley Y (2017) First human images from a next generation SiPM based PET/CT system with improved time and spatial resolution. Eur J Nucl Med Mol Imaging 44(Suppl. 2):S303

16. Lecoq P (2017) Pushing the limits in time-of-flight PET imaging. IEEE Trans Radiat Plas Med Sci 1(6):473-485. https://doi. org/10.1109/TRPMS.2017.2756674

17. Haemisch Y, Frach T, Degenhardt C, Thon A (2012) Fully digital arrays of silicon photomultipliers (dSiPM) - a scalable alternative to vacuum photomultiplier tubes (PMT). Phys Procedia 37(Supplement C):1546-1560. https://doi.org/10.1016/j.phpro .2012 .03 .749

18. Liu Z, Pizzichemi M, Auffray E, Lecoq P, Paganoni M (2016) Performance study of Philips digital silicon photomultiplier coupled to scintillating crystals. J Instrum 11(01):P01017

19. Krishnamoorthy S, Schmall JP, Surti S (2017) PET physics and instrumentation. In: Khalil MM (ed) Basic science of PET imaging. Springer, Heidelberg

20. Saoudi A, Pepin CM, Dion F, Bentourkia M, Lecomte R, Andreaco M, Casey M, Nutt R, Dautet $H$ (1999) Investigation of depth-of-interaction by pulse shape discrimination in multicrystal detectors read out by avalanche photodiodes. IEEE Trans Nucl Sci 46(3):462-467. https://doi.org/10.1109/23.775563

21. Seidel J, Vaquero JJ, Siegel S, Gandler WR, Green MV (1999) Depth identification accuracy of a three layer phoswich PET detector module. IEEE Trans Nucl Sci 46(3):485-490. https:// doi.org/10.1109/23.775567

22. Miyaoka RS, Lewellen TK, Yu H, McDaniel DL (1998) Design of a depth of interaction (DOI) PET detector module. IEEE Trans Nucl Sci 45(3):1069-1073. https://doi.org/10.1109/23.681980

23. de Jong HW, van Velden FH, Kloet RW, Buijs FL, Boellaard R, Lammertsma AA (2007) Performance evaluation of the ECAT HRRT: an LSO-LYSO double layer high resolution, high sensitivity scanner. Phys Med Biol 52(5):1505-1526. https://doi. org/10.1088/0031-9155/52/5/019

24. Moses WW, Derenzo SE, Melcher CL, Manente RA (1995) A room temperature LSO/PIN photodiode PET detector module that measures depth of interaction. IEEE Trans Nucl Sci 42(4):1085-1089. https://doi.org/10.1109/23.467744

25. Dokhale PA, Silverman RW, Shah KS, Grazioso R, Farrell R, Glodo J, McClish MA, Entine G, Tran VH, Cherry SR (2004) Performance measurements of a depth-encoding PET detector module based on position-sensitive avalanche photodiode readout. Phys Med Biol 49(18):4293-4304

26. Burr KC, Ivan A, Castleberry DE, LeBlanc JW, Shah KS, Farrell R (2004) Evaluation of a prototype small-animal PET detector with depth-of-interaction encoding. IEEE Trans Nucl Sci 51(4):1791-1798. https://doi.org/10.1109/TNS.2004.83290 5

27. Yamaya T, Mitsuhashi T, Matsumoto T, Inadama N, Nishikido F, Yoshida E, Murayama H, Kawai H, Suga M, Watanabe M (2011) A SiPM-based isotropic-3D PET detector X'tal cube with a three-dimensional array of $1 \mathrm{~mm}(3)$ crystals. Phys Med Biol 56(21):6793-6807. https://doi.org/10.1088/0031-9155/56/21/003

28. Lee MS, Kim KY, Ko GB, Lee JS (2017) Prototype pre-clinical PET scanner with depth-of-interaction measurements using single-layer crystal array and single-ended readout. Phys Med Biol 62(10):3983-3996. https://doi.org/10.1088/1361-6560/aa64c7

29. Zhang Y, Yan H, Baghaei H, Wong WH (2016) A novel depthof-interaction block detector for positron emission tomography using a dichotomous orthogonal symmetry decoding concept. Phys Med Biol 61(4):1608-1633. https://doi. org/10.1088/0031-9155/61/4/1608

30. Pizzichemi M, Stringhini G, Niknejad T, Liu Z, Lecoq P, Tavernier S, Varela J, Paganoni M, Auffray E (2016) A new method for depth of interaction determination in PET detectors. Phys Med Biol 61(12):4679

31. Clinthorne NH, Rogers WL, Shao L, Koral KF (1987) A hybrid maximum likelihood position computer for scintillation cameras. IEEE Trans Nucl Sci 34(1):97-101. https://doi.org/10.1109/ TNS.1987.4337309

32. Jinhun J, Miyaoka RS, Kohlmyer S, Lewellen TK (2000) Implementation of ML based positioning algorithms for scintillation cameras. IEEE Trans Nucl Sci 47(3):1104-1111. https://doi. org/10.1109/23.856555

33. Gagnon D, Pouliot N, Laperriere L, Therrien M, Olivier P (1993) Maximum likelihood positioning in the scintillation camera using depth of interaction. IEEE Trans Med Imaging 12(1):101-107. https://doi.org/10.1109/42.222673

34. Hunter WCJ, Barrett HH, Furenlid LR (2009) Calibration method for ML estimation of 3D interaction position in a thick gammaray detector. IEEE Trans Nucl Sci 56(1):189-196. https://doi. org/10.1109/TNS.2008.2010704

35. Ling T, Lewellen TK, Miyaoka RS (2007) Depth of interaction decoding of a continuous crystal detector module. Phys Med Biol 52(8):2213-2228. https://doi.org/10.1088/0031-9155/52/8/012

36. Maas MC, Schaart DR, van der Laan DJ, Bruyndonckx P, Lemaitre C, Beekman FJ, van Eijk CW (2009) Monolithic scintillator PET detectors with intrinsic depth-of-interaction correction. Phys Med Biol 54(7):1893-1908. https://doi. org/10.1088/0031-9155/54/7/003

37. van Dam HT, Seifert S, Vinke R, Dendooven P, Lohner H, Beekman FJ, Schaart DR (2011) A practical method for depth of interaction determination in monolithic scintillator PET detectors. Phys Med Biol 56(13):4135-4145. https://doi. org/10.1088/0031-9155/56/13/025

38. Pedemonte S, Pierce L, Van Leemput K (2017) A machine learning method for fast and accurate characterization of depthof-interaction gamma cameras. Phys Med Biol. https://doi. org/10.1088/1361-6560/aa6ee5

39. Heller S, Zanzonico P (2011) Nuclear probes and intraoperative gamma cameras. Semin Nucl Med 41(3):166-181. https://doi. org/10.1053/j.semnuclmed.2010.12.004 
40. Lees JE, Bassford DJ, Blake OE, Blackshaw PE, Perkins AC (2012) A hybrid camera for simultaneous imaging of gamma and optical photons. J Instrum 7(06):P06009

41. O'Connor M, Rhodes D, Hruska C (2009) Molecular breast imaging. Expert Rev Anticancer Ther 9(8):1073-1080. https ://doi.org/10.1586/era.09.75

42. Rhodes DJ, Hruska CB, Phillips SW, Whaley DH, O'Connor MK (2011) Dedicated dual-head gamma imaging for breast cancer screening in women with mammographically dense breasts. Radiology 258(1):106-118. https://doi.org/10.1148/ radiol.10100625

43. Erlandsson K, Kacperski K, van Gramberg D, Hutton BF (2009) Performance evaluation of D-SPECT: a novel SPECT system for nuclear cardiology. Phys Med Biol 54(9):26352649. https://doi.org/10.1088/0031-9155/54/9/003

44. Gambhir SS, Berman DS, Ziffer J, Nagler M, Sandler M, Patton J, Hutton B, Sharir T, Haim SB, Haim SB (2009) A novel highsensitivity rapid-acquisition single-photon cardiac imaging camera. J Nucl Med 50(4):635-643. https://doi.org/10.2967/ jnumed.108.060020

45. Bocher M, Blevis IM, Tsukerman L, Shrem Y, Kovalski G, Volokh L (2010) A fast cardiac gamma camera with dynamic SPECT capabilities: design, system validation and future potential. Eur J Nucl Med Mol Imaging 37(10):1887-1902. https://doi.org/10.1007/s00259-010-1488-Z

46. Esteves FP, Raggi P, Folks RD, Keidar Z, Askew JW, Rispler S, O'Connor MK, Verdes L, Garcia EV (2009) Novel solidstate-detector dedicated cardiac camera for fast myocardial perfusion imaging: multicenter comparison with standard dual detector cameras. J Nucl Cardiol 16(6):927-934. https://doi. org/10.1007/s12350-009-9137-2

47. Barrett HH, Furenlid LR, Freed M, Hesterman JY, Kupinski MA, Clarkson E, Whitaker MK (2008) Adaptive SPECT. IEEE Trans Med Imaging 27(6):775-788. https://doi.org/10.1109/ TMI.2007.913241

48. Brzymialkiewicz CN, Tornai MP, McKinley RL, Bowsher JE (2005) Evaluation of fully 3-D emission mammotomography with a compact cadmium zinc telluride detector. IEEE Trans Med Imaging 24(7):868-877. https://doi.org/10.1109/ TMI.2005.852501

49. Beekman FJ, van der Have F, Goorden MC, Vaissier PEB, van Roosmalen J, During H, Vastenhouw B (2015) G-SPECT-I: a full ring high sensitivity and ultra-fast clinical molecular imaging system with $<3 \mathrm{~mm}$ resolution. Eur J Nucl Med Mol Imaging 42:S209

50. Rogulski MM, Barber HB, Barrett HH, Shoemaker RL, Woolfenden JM (1993) Ultra-high-resolution brain SPECT imaging: simulation results. IEEE Trans Nucl Sci 40(4):11231129. https://doi.org/10.1109/23.256722

51. Goorden MC, Rentmeester MC, Beekman FJ (2009) Theoretical analysis of full-ring multi-pinhole brain SPECT. Phys Med Biol 54(21):6593-6610. https://doi. org/10.1088/0031-9155/54/21/010

52. Metzler SD, Accorsi R, Novak JR, Ayan AS, Jaszczak RJ (2006) On-axis sensitivity and resolution of a slit-slat collimator. J Nucl Med 47(11):1884-1890

53. Busca P, Fiorini C, Occhipinti M, Trigilio P, Nagy K, Bükki T, Czeller M, Nyitrai Z, Piemonte C, Ferri A, Gola A, Rieger J (2015) A SiPM-based detection module for SPECT/MRI systems. In: 2015 IEEE nuclear science symposium and medical imaging conference (NSS/MIC), Oct. 31 2015-Nov. 7 2015, pp 1-3. https://doi.org/10.1109/nssmic.2015.7582257

54. Salvado D, Erlandsson K, Bousse A, Occhipinti M, Busca $\mathrm{P}$, Fiorini C, Hutton BF (2015) Collimator design for a brain SPECT/MRI insert. IEEE Trans Nucl Sci 62(4):1716-1724. https ://doi.org/10.1109/TNS.2015.2450017
55. Hutton BF, Occhipinti M, Kuehne A, Mathe D, Kovacs N, Waiczies H, Erlandsson K, Salvado D, Carminati M, Montagnani GL, Short SC, Ottobrini L, van Mullekom P, Piemonte C, Bukki T, Nyitrai Z, Papp Z, Nagy K, Niendorf T, de Francesco I, Fiorini C, Consortium I (2017) Development of clinical simultaneous SPECT/MRI. Brit J Radiol. https://doi.org/10.1259/bjr.20160690

56. Deprez K, Vandenberghe S, Van Audenhaege K, Van Vaerenbergh J, Van Holen R (2013) Rapid additive manufacturing of MR compatible multipinhole collimators with selective laser melting of tungsten powder. Med Phys 40(1):1-11

57. Van Audenhaege K, Van Hole R, Vandenberghe S, Vanhove C, Metzler SD, Moore SC (2015) Review of SPECT collimator selection, optimization, and fabrication for clinical and preclinical imaging. Med Phys 42:4796-4813

58. González AJ, Majewski S, Sánchez F, Aussenhofer S, Aguilar A, Conde P, Hernández L, Vidal LF, Pani R, Bettiol M, Fabbri A, Bert J, Visvikis D, Jackson C, Murphy J, O’Neill K, Benlloch JM (2016) The MINDView brain PET detector, feasibility study based on SiPM arrays. Nucl Instr Meth Phys Res A 818(Supplement C):82-90. https://doi.org/10.1016/j.nima.2016.02.046

59. Melroy S, Bauer C, McHugh M, Carden G, Stolin A, Majewski S, Brefczynski-Lewis J, Wuest T (2017) Development and design of next-generation head-mounted ambulatory microdose positronemission tomography (AM-PET) system. Sensors (Basel, Switzerland) 17(5):1164. https://doi.org/10.3390/s17051164

60. Schmidtlein CR, Turner JN, Thompson MO, Mandal KC, Häggström I, Zhang J, Humm JL, Feiglin DH, Krol A (2016) Performance modeling of a wearable brain PET (BET) camera. In: SPIE medical imaging. SPIE, p 11

61. Yamamoto S, Honda M, Oohashi T, Shimizu K, Senda M (2011) Development of a brain PET system, PET-Hat: a wearable PET system for brain research. IEEE Trans Nucl Sci 58(3):668-673. https://doi.org/10.1109/TNS.2011.2105502

62. Martins MV (2015) Positron emission mammography, mammography techniques and review. In: Fernandes F (ed) InTech. https ://doi.org/10.5772/60452

63. Neves JA (2011) The ClearPEM breast imaging scanner. Nucl Instr Meth A 628:444-447

64. Lee E, Werner ME, Karp JS, Surti S (2013) Design optimization of a time-of-flight, breast PET scanner. IEEE Trans Nucl Sci 60(3):1645-1652. https://doi.org/10.1109/TNS.2013.2257849

65. Tashima H, Yamaya $T$ (2013) Impact of TOF information in OpenPET imaging. In: 2013 IEEE nuclear science symposium and medical imaging conference (2013 NSS/MIC), Oct. 27 2013-Nov. 2 2013, pp 1-3. https://doi.org/10.1109/nssmi c. 2013.6829340

66. Mawlawi O, Townsend DW (2009) Multimodality imaging: an update on PET/CT technology. Eur J Nucl Med Mol Imaging 36(Suppl 1):S15-S29. https://doi.org/10.1007/s0025 9-008-1016-6

67. Pichler BJ, Judenhofer MS, Wehrl HF (2008) PET/MRI hybrid imaging: devices and initial results. Europ Radiol 18(6):10771086. https://doi.org/10.1007/s00330-008-0857-5

68. Delso G, Furst S, Jakoby B, Ladebeck R, Ganter C, Nekolla SG, Schwaiger M, Ziegler SI (2011) Performance measurements of the Siemens mMR integrated whole-body PET/MR scanner. J Nucl Med 52(12):1914-1922. https://doi.org/10.2967/jnume d.111.092726

69. Vandenberghe S, Marsden PK (2015) PET-MRI: a review of challenges and solutions in the development of integrated multimodality imaging. Phys Med Biol 60(4):R115-R154. https:// doi.org/10.1088/0031-9155/60/4/R115

70. Miller M, Zhang J, Binzel K, Griesmer J, Laurence T, Marayan M, Natarajamani D, Wang S, Knopp M (2015) Characterization of the vereos digital photon counting PET system. J Nucl Med 56(Suppl. 3):434 
71. Hsu DFC, Ilan E, Peterson WT, Uribe J, Lubberink M, Levin CS (2017) Studies of a next-generation silicon-photomultiplierbased time-of-flight PET/CT system. J Nucl Med 58(9):15111518. https://doi.org/10.2967/jnumed.117.189514

72. Lois C, Jakoby BW, Long MJ, Hubner KF, Barker DW, Casey ME, Conti M, Panin VY, Kadrmas DJ, Townsend DW (2010) An assessment of the impact of incorporating time-of-flight information into clinical PET/CT imaging. J Nucl Med 51(2):237-245. https://doi.org/10.2967/jnumed.109.068098

73. Ladefoged CN, Law I, Anazodo U, St Lawrence K, IzquierdoGarcia D, Catana C, Burgos N, Cardoso MJ, Ourselin S, Hutton B, Merida I, Costes N, Hammers A, Benoit D, Holm S, Juttukonda M, An H, Cabello J, Lukas M, Nekolla S, Ziegler S, Fenchel M, Jakoby B, Casey ME, Benzinger T, Hojgaard L, Hansen AE, Andersen FL (2017) A multi-centre evaluation of eleven clinically feasible brain PET/MRI attenuation correction techniques using a large cohort of patients. Neuroimage 147:346-359. https://doi.org/10.1016/j.neuroimage .2016.12.010

74. Cherry SR, Badawi RD, Karp JS, Moses WW, Price P, Jones T (2017) Total-body imaging: transforming the role of positron emission tomography. Sci Transl Med. https://doi.org/10.1126/ scitranslmed.aaf6169

75. Qi J, Leahy RM (2006) Iterative reconstruction techniques in emission computed tomography. Phys Med Biol 51(15):R541R578. https://doi.org/10.1088/0031-9155/51/15/R01

76. Hudson HM, Larkin RS (1994) Accelerated image reconstruction using ordered subsets of projection data. IEEE Trans Med Imaging 13(4):601-609. https://doi.org/10.1109/42.363108

77. Parra L, Barrett HH (1998) List-mode likelihood: EM algorithm and image quality estimation demonstrated on 2-D PET. IEEE Trans Med Imaging 17(2):228-235. https://doi. org/10.1109/42.700734

78. Reader AJ, Erlandsson K, Flower MA, Ott RJ (1998) Fast accurate iterative reconstruction for low-statistics positron volume imaging. Phys Med Biol 43(4):835-846

79. Nuyts J, Beque D, Dupont P, Mortelmans L (2002) A concave prior penalizing relative differences for maximum-a-posteriori reconstruction in emission tomography. IEEE Trans Nucl Sci 49(1):56-60. https://doi.org/10.1109/TNS.2002.998681

80. Teoh EJ, McGowan DR, Macpherson RE, Bradley KM, Gleeson FV (2015) Phantom and clinical evaluation of the bayesian penalized likelihood reconstruction algorithm Q.Clear on an LYSO PET/CT system. J Nucl Med 56(9):1447-1452. https:// doi.org/10.2967/jnumed.115.159301

81. Yang L, Zhou J, Ferrero A, Badawi RD, Qi J (2014) Regularization design in penalized maximum-likelihood image reconstruction for lesion detection in 3D PET. Phys Med Biol 59(2):403-419. https://doi.org/10.1088/0031-9155/59/2/403

82. Bai B, Li Q, Leahy RM (2013) Magnetic resonance-guided positron emission tomography image reconstruction. Semin Nucl Med 43(1):30-44. https://doi.org/10.1053/j.semnuclmed .2012 .08 .006

83. Erlandsson K, Dickson J, Arridge S, Atkinson D, Ourselin S, Hutton BF (2016) MR imaging-guided partial volume correction of pet data in PET/MR imaging. PET Clin 11(2):161-177. https://doi.org/10.1016/j.cpet.2015.09.002

84. Erlandsson K, Buvat I, Pretorius PH, Thomas BA, Hutton BF (2012) A review of partial volume correction techniques for emission tomography and their applications in neurology, cardiology and oncology. Phys Med Biol 57(21):R119-R159. https://doi.org/10.1088/0031-9155/57/21/R119

85. Walrand S, Hesse M, Jamar F (2016) Update on novel trends in PET/CT technology and its clinical applications. Brit J Radiol. https://doi.org/10.1259/bjr.20160534
86. Ljungberg M, Pretorius PH (2017) SPECT/CT: an update on technological developments and clinical applications. Brit $\mathrm{J}$ Radiol. https://doi.org/10.1259/bjr.20160402

87. Cabello J, Ziegler SI (2016) Advances in PET/MR instrumentation and image reconstruction. Brit J Radiol. https://doi. org/10.1259/bjr.20160363

88. Ehrhardt MJ, Thielemans K, Pizarro L, Atkinson D, Ourselin S, Hutton BF, Arridge SR (2015) Joint reconstruction of PET-MRI by exploiting structural similarity. Inv Probl 31(1):015001

89. Conti M (2011) Why is TOF PET reconstruction a more robust method in the presence of inconsistent data? Phys Med Biol 56(1):155-168. https://doi.org/10.1088/0031-9155/56/1/010

90. Ahn S, Cheng L, Manjeshwar RM (2014) Analysis of the effects of errors in attenuation maps on PET quantitation in TOF PET. In: 2014 IEEE nuclear science symposium and medical imaging conference (NSS/MIC), 8-15 Nov. 2014, pp 1-4. https://doi. org/10.1109/nssmic.2014.7430781

91. Mehranian A, Zaidi H (2015) Impact of time-of-flight PET on quantification errors in MR imaging-based attenuation correction. J Nucl Med 56(4):635-641. https://doi.org/10.2967/jnume d.114.148817

92. Defrise M, Rezaei A, Nuyts J (2012) Time-of-flight PET data determine the attenuation sinogram up to a constant. Phys Med Biol 57(4):885-899. https://doi.org/10.1088/0031-9155/57/4/885

93. Rezaei A, Defrise M, Nuyts J (2014) ML-reconstruction for TOF-PET with simultaneous estimation of the attenuation factors. IEEE Trans Med Imaging 33(7):1563-1572. https://doi. org/10.1109/TMI.2014.2318175

94. Qi W, Yang Y, Song C, Wernick MN, Pretorius PH, King MA (2017) 4-D reconstruction with respiratory correction for gated myocardial perfusion SPECT. IEEE Trans Med Imaging 36(8):1626-1635. https://doi.org/10.1109/TMI.2017.2690819

95. Feng T, Wang J, Fung G, Tsui B (2016) Non-rigid dual respiratory and cardiac motion correction methods after, during, and before image reconstruction for 4D cardiac PET. Phys Med Biol 61(1):151-168. https://doi.org/10.1088/0031-9155/61/1/151

96. Bousse A, Bertolli O, Atkinson D, Arridge S, Ourselin S, Hutton BF, Thielemans K (2016) Maximum-likelihood joint image reconstruction/motion estimation in attenuation-corrected respiratory gated PET/CT using a single attenuation map. IEEE Trans Med Imaging 35(1):217-228. https://doi.org/10.1109/ TMI.2015.2464156

97. Erlandsson K (2011) tracer kinetic models: basics and concepts. In: Khalil MM (ed) Basic sciences of nuclear medicine. Springer, Heidelberg, pp 333-351

98. Bentourkia M (2011) Tracer kinetic models: methodology and applications. In: Khalil MM (ed) Basic sciences of nuclear medicine. Springer, Heidelberg, pp 353-376

99. Carson RE, Lange K (1985) The EM parametric image reconstruction algorithm. J Am Stat Assoc 80(389):20-22. https://doi. org/10.2307/2288031

100. Tsoumpas C, Turkheimer FE, Thielemans K (2008) A survey of approaches for direct parametric image reconstruction in emission tomography. Med Phys 35(9):3963-3971. https://doi. org/10.1118/1.2966349

101. Rahmim A, Tang J, Zaidi H (2009) Four-dimensional (4D) image reconstruction strategies in dynamic PET: beyond conventional independent frame reconstruction. Med Phys 36(8):3654-3670. https://doi.org/10.1118/1.3160108

102. Gullberg GT, Reutter BW, Sitek A, Maltz JS, Budinger TF (2010) Dynamic single photon emission computed tomography-basic principles and cardiac applications. Phys Med Biol 55(20):R111R191. https://doi.org/10.1088/0031-9155/55/20/R01

103. Reader AJ, Verhaeghe J (2014) 4D image reconstruction for emission tomography. Phys Med Biol 59(22):R371-R418. https ://doi.org/10.1088/0031-9155/59/22/R371 
104. Jiao J, Bousse A, Thielemans K, Burgos N, Weston PS, Schott JM, Atkinson D, Arridge SR, Hutton BF, Markiewicz P, Ourselin S (2017) Direct parametric reconstruction with joint motion estimation/correction for dynamic brain PET data. IEEE Trans Med Imaging 36(1):203-213. https://doi.org/10.1109/ TMI.2016.2594150

105. Erlandsson K, Liljeroth M, Atkinson D, Arridge S, Ourselin S, Hutton BF (2016) Improved parameter-estimation with MRIconstrained PET kinetic modeling: a simulation study. IEEE Trans Nucl Sci 63(5):2464-2470. https://doi.org/10.1109/ TNS.2015.2507444

106. Sari H, Erlandsson K, Law I, Larsson HB, Ourselin S, Arridge S, Atkinson D, Hutton BF (2017) Estimation of an image derived input function with MR-defined carotid arteries in FDG-PET human studies using a novel partial volume correction method. J Cereb Blood Flow Metab 37(4):1398-1409. https://doi. org/10.1177/0271678X16656197

107. Wells RG, Timmins R, Klein R, Lockwood J, Marvin B, deKemp RA, Wei L, Ruddy TD (2014) Dynamic SPECT measurement of absolute myocardial blood flow in a porcine model. J Nucl Med $55: 1-7$

108. Markiewicz PJ, Thielemans K, Schott JM, Atkinson D, Arridge SR, Hutton BF, Ourselin S (2016) Rapid processing of PET list-mode data for efficient uncertainty estimation and data analysis. Phys Med Biol 61(13):N322-N336. https://doi. org/10.1088/0031-9155/61/13/N322 